\title{
Efectividad del programa "IVAA-SCHILLER" en prevención secundaria de cáncer cervical en estudiantes de obstetricia Universidad Roosevelt, Huancayo-2021
} Effectiveness of the "IVAA-SCHILLER" program in secondary prevention of cervical cancer in obstetric
students, Roosevelt University Huancayo-2021

Liz Miriam Suárez Reynoso ${ }^{1, a}$, Javier Juan Aliaga Salguero ${ }^{1, b}$.

\section{RESUMEN}

Objetivos: Determinar la influencia del programa "IVAA-SCHILLER" en prevención secundaria de cáncer cervical en estudiantes del VII y VIII semestre de la Escuela Profesional de Obstetricia de la Universidad Privada de Huancayo Franklin Roosevelt-2021. Material y métodos: se consideró el método científico, de tipo aplicada, con diseño preexperimental con pre y post test, la población estuvo conformada por 80 estudiantes del VII y VIII semestre; la muestra fue de 57 estudiantes determinada por conveniencia; como técnica la encuesta y como instrumento el cuestionario, los Resultados: de la prueba de entrada, se observó que el 8,8\% de los estudiantes lograron el puntaje de 6 , el 1,8\% alcanzaron puntajes de 7 y 15 , además el $12,3 \%$ lograron el puntaje de 8 , el 15,8\% lograron el puntaje de 9 , el $21,1 \%$ alcanzaron el puntaje de 10 , el 3,5\% alcanzaron puntajes de 13 y 14 respectivamente; con respecto a la prueba de salida, el $17,5 \%$ lograron puntajes de 10,11 y 15 , el $36,8 \%$ obtuvieron puntaje de 12 , el 10,5\% lograron el puntaje de 14. Conclusiones: existe diferencia estadísticamente significativa de promedios en la población entre la prueba de entrada y prueba de salida; por lo tanto; se acepta la hipótesis alterna (Ha).

PALABRAS CLAVE: prevención secundaria de cáncer cervical, test de IVAA, test de Schiller.

\section{SUMMARY}

Objective: To determine the influence of the "IVAA-SCHILLER" program in secondary prevention of cervical cancer en students of the VII and VIII semester of the Professional School of Obstetrics of the Private University of Huancayo Franklin Roosevelt-2021. Material and methods: the scientific method was considered, of an applied type, with a pre-experimental design with pre and post test, the population consisted of 80 students from the VII and VIII semester; the sample was of 57 students determined by convenience, As a technique the survey and as an instrument the questionnaire. Results: of the entrance test, it was observed that $8.8 \%$ of the students achieved a score of $6,1.8 \%$ achieved scores of 7 and 15 , in addition to the $12.3 \%$ achieved a score of $8,15.8 \%$ achieved score of 13 and 14 respectively; Regarding the exit test, $17.5 \%$ achieved scores of 10,1 and $15,36.8 \%$ obtained a score of 2 , $10.5 \%$ achieved a score of 14. Conclusions: there is a statistically significant difference in averages I the population between the entry trial and exit trial; Thus; the alternative Hypothesis (Ha) is accepted.

KEYWORDS: secondary prevention of cervical cancer, IVAA test, Schiller test.

\footnotetext{
Universidad Privada de Huancayo Franklin Roosevelt Huancayo. Perú

Docente; Médico; Magister. ORCID ID: 0000-0001-9723-2040

Docente; Médico; Magister. ORCID ID: 0000-0003-3349-7175
} 


\section{INTRODUCCIÓN}

El cérvix o cuello uterino, parte inferior del útero que forma parte del aparato reproductor interno femenino; el cáncer cervical patología frecuente en nuestro medio y considerado como prioridad del Ministerio de Salud ya que la implementación de otros métodos como screening a través de la aplicación de ácido acético llamado test de IVAA; o la aplicación del lugol llamado test de Schiller o test de IVIL. El cáncer de cérvix es una patología prevenible y que es considerado una de las principales causa de muerte por cáncer en la mujer mundialmente y se presenta con más frecuencia en países de ingresos bajos y medianos y cuyo agente causal es el papiloma virus humano de alto riesgo contraída a través del contacto sexual. El cáncer cervical es frecuente en 45 países dentro de ellos tenemos a África, Asia y algunos países de Centro y Sudamérica (1).

Cáncer de cuello uterino patología de fácil diagnóstico y en gran parte prevenible; el agente etiológico es el Virus del Papiloma Humano adquirido durante el contacto sexual con una persona infectada y ocupa el cuarto lugar en incidencia y mortalidad con 270,000 muertes por año según Globocan 2018 (2).

Por ello el cáncer cervical es considerado un problema de salud pública y aún más álgido por el diagnóstico tardio como consecuencia de las debilidades del sistema de salud, temores de la población que conllevan a costos altos de tratamiento y con resultados sub óptimos con impacto negativo para la supervivencia de las pacientes (3).

Como procedimiento alternativo al Papanicolaou podemos mencionar a la inspección visual con el ácido acético y la inspección visual con el lugol; que se puede realizar en establecimientos de primer nivel, en lugares de difícil accesibilidad y en donde no se cuente con el apoyo de laboratorios de citología, realizado por profesional debidamente capacitado (obstetra) $(3,4)$.

Para Abanto, que realizó un estudio sobre inspección visual con ácido acético y Papanicolaou en la detección de lesiones intraepitelial de alto grado del cuello uterino en pacientes de Cuídate consultorios especializados - La Victoria; concluyendo que: La inspección visual con al ácido acético en una alternativa muy buena para el tamizado del cérvix debido a que la sensibilidad es superior a la citología sin embargo se debe tomar en consideración los falsos positivos que podría conllevar a tratamientos que no son necesarios (5).

Para Bellido en su investigación titulada Conocimientos adquiridos durante el año de internado sobre prevención secundaria del cáncer de cuello uterino en estudiantes universitarios de la Escuela Académico Profesional de Obstetricia, Año 2014 tesis hecha en la Universidad Nacional Mayor de San Marcos, el estudio que realizaron fue de tipo observacional, descriptivo comparativo, de corte transversal y prospectivo; llegando a concluir que el nivel de conocimiento adquirido durante el internado sobre prevención secundaria del cáncer de cuello uterino en estudiantes universitarios fue medio al inicio del internado con $49,2 \%$ y llegando a $52,8 \%$ al terminar el internado de tal manera que hubo un aumento de $3,6 \%$ (6).

Para Del Valle A. en su investigación Inspección visual con ácido acético (IVAA) en la detección precoz del cáncer de cuello uterino - estudio comparativo tesis realizada en la Universidad Nacional de Córdoba - Argentina para optar el grado académico de Doctor en Medicina y Cirugía; el estudio fue transversal en donde los residentes apropiadamente capacitados tomaron muestras de Papanicolaou simultáneamente realizaron la IVAA, Schiller y la colposcopia en un total de 2230 damas que acudieron espontáneamente a los consultorios de ginecología del Hospital Nacional de Clínicas y la clínica de Sanagec de Córdoba, excluyendo en esta investigación a damas que tuvieron tratamiento de lesiones neoplásicas, grávidas y con alteraciones en la anatomía de la parte cervical y uterina; concluyendo que: La elevada sensibilidad de la IVAA demuestra que la prueba podría ser de muchas utilidad para la detección de las lesiones precancerosas del cérvix ya sea como método de pesquisa asociado además al Papanicolaou; por otro lado al obtener los resultados de forma inmediata se podría evitar la deserción relacionado al tratamiento y seguimiento en mujeres con sospecha de patología del cérvix de esa forma el manejo seria el correcto y por lo tanto se disminuiría la prevalencia de esta enfermedad (7).

\section{La Organización Mundial de la Salud define prevención en tres niveles como son:}

La prevención primaria, prevención secundaria con el diagnóstico precoz y tratamiento oportuno o ver y tratar, y la prevención terciaria (8). 
El tamizaje y la detección precoz nos permite considerar el enfoque "Ver y Tratar", de esa forma estaríamos resolviendo el problema. Por otro lado, se considera también que después de haber realizado el tamizaje como pap o IVAA con resultados negativos, el próximo control será en tres años, por otro lado, si se realizó tamizaje de $\mathrm{PVH}$, con resultado negativo el próximo tamizaje debe ser en un periodo no menor de 5 años. El tamizaje de cérvix se recomienda a partir de los 30 años. Se demuestra en datos recientes que la IVAA es tan eficaz que el Papanicolaou. Se menciona también que en el año 94 se hizo un estudio en Sudáfrica en el cual el valor predictivo positivo fue similar tanto en el IVAA como en el pap; por otro lado otros estudios hechos en la India mencionaron que la inspección visual con ácido acético es alterativa viable para el pap como una prueba primeria de tamizaje; considerando también que la prueba con ácido acético tiene una técnica de fácil aprestamiento, de bajo costo y muy sensible ideal para países en desarrollo y para lugares de bajos recursos o poca accesibilidad, y la clave para el desempeño adecuada en esta área es la capacitación del personal para la realización de la misma (9).

\section{MATERIAL Y MÉTODOS}

La presente investigación es de enfoque cuantitativo con diseño pre experimental y de corte longitudinal; la población estuvo conformada por 80 estudiantes del VII y VIII semestres de la Escuela Profesional de Obstetricia; y la muestra fue de 57; para la recolección de datos se consideró como técnica la encuesta y como instrumento el cuestionario validado a través de juicio de expertos y la confiabilidad por alfa de Cronbach con 0.89; del mismo modo se utilizó la tabla de frecuencias y porcentajes; del mismo modo se utilizó también estadísticos para el pre y post test; para la prueba de hipótesis de se utilizó el estadígrafo t de Student y para la prueba de normalidad a Kolmogorov-Smirnov para una muestra.

\section{RESULTADOS}

Con respecto a la prueba de entrada acerca de prevención secundaria de cáncer cervical en los estudiantes del VII y VIII semestre de la Escuela Profesional de Obstetricia, se observó que el 8,8\% de los estudiantes en la prueba de entrada lograron el puntaje de 6, como también el 1,8\% de los estudiantes alcanzaron los puntajes de 7 y 15, además el 12,3\% de los estudiantes lograron el puntaje de 8 , así mismo el $15,8 \%$ de los estudiantes lograron el puntaje de 9 , por otro lado el $21,1 \%$ de los estudiantes alcanzaron el puntaje de 10, de igual forma el 3,5\% de los estudiantes alcanzaron los puntajes de 13 y 14 respectivamente (tabla 1 y gráfico 1).

Con respecto a la prueba de salida acerca de prevención secundaria de cáncer cervical en los estudiantes del VII y VIII semestre de la Escuela Profesional de Obstetricia, se observó que el 17,5\% de los estudiantes en la prueba de salida lograron los puntajes de 10, 11 y 15, como también el $36,8 \%$ de

Tabla 1. Frecuencia de la prueba de entrada acerca de prevención secundaria

\begin{tabular}{llllll}
\hline & & Frecuencia & Porcentaje & $\begin{array}{l}\text { Porcentaje } \\
\text { válido }\end{array}$ & $\begin{array}{l}\text { Porcentaje } \\
\text { acumulado }\end{array}$ \\
\hline 6.00 & 5 & 8,8 & 8,8 & 8,8 \\
& 7.00 & 1 & 1,8 & 1,8 & 10,5 \\
& 8.00 & 7 & 12,3 & 12,3 & 22,8 \\
Válido & 9 & 15,8 & 15,8 & 38,6 \\
& 9.00 & 12 & 21,1 & 21,1 & 59,6 \\
& 10.00 & 17,5 & 17,5 & 77,2 \\
& 11.00 & 10 & 14,0 & 14,0 & 91,2 \\
& 12.00 & 8 & 3,5 & 3,5 & 94,7 \\
& 13.00 & 2 & 3,5 & 3,5 & 98,2 \\
& 14.00 & 2 & 1,8 & 1,8 & 100,0 \\
\hline Fuente. Base de datos de la prueba de entrada del cuestionario aplicado & &
\end{tabular}




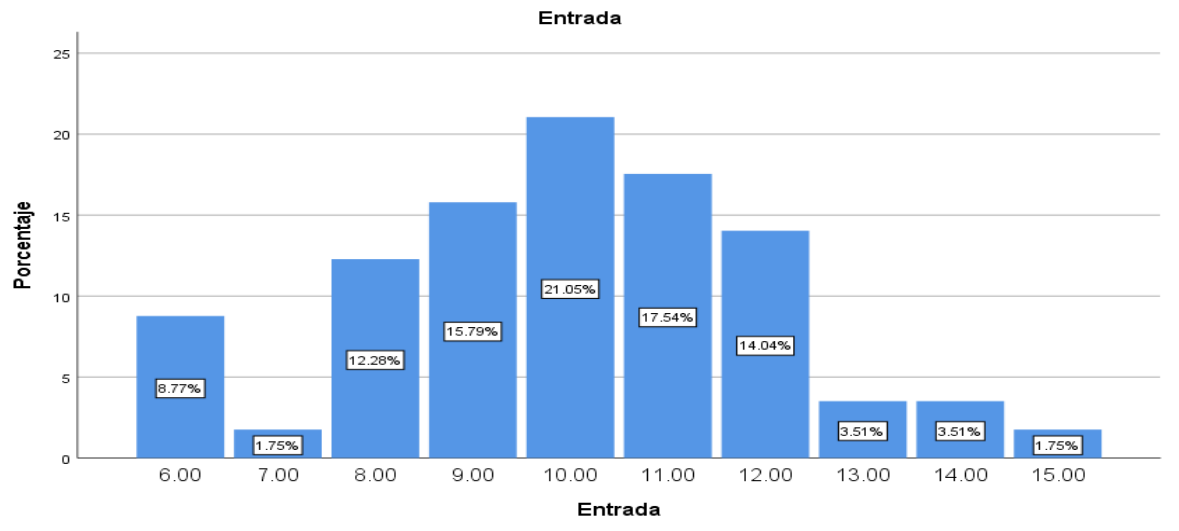

Fuente. Base de datos de la prueba de entrada del cuestionario aplicado Gráfico 1. Porcentaje de la prueba de entrada

Tabla 2. Frecuencia de la prueba de salida acerca de prevención secundaria

Salida

\begin{tabular}{cccccc}
\hline & & Frecuencia & Porcentaje & $\begin{array}{c}\text { Porcentaje } \\
\text { válido }\end{array}$ & $\begin{array}{c}\text { Porcentaje } \\
\text { acumulado }\end{array}$ \\
& 10.00 & 10 & 17,5 & 17,5 & 17,5 \\
\hline \multirow{4}{*}{ Válido } & 11.00 & 10 & 17,5 & 17,5 & 35,1 \\
& 12.00 & 21 & 36,8 & 36,8 & 71,9 \\
& 14.00 & 10 & 17,5 & 17,5 & 89,5 \\
& 15.00 & 6 & 10,5 & 10,5 & 100,0 \\
& Total & 57 & 100,0 & 100,0 & \\
\hline
\end{tabular}

Fuente. Base de datos de la prueba de salida del cuestionario aplicado

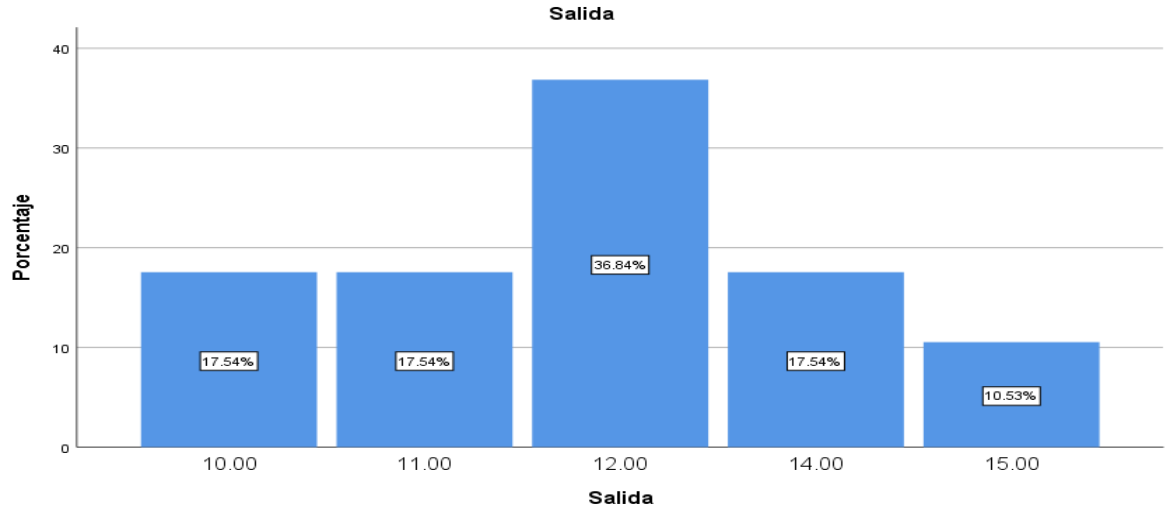

Fuente. Base de datos de la prueba de salida del cuestionario aplicado

Gráfico 2. Porcentaje de la prueba de salida

los estudiantes alcanzaron el puntaje de 12 , además el $10,5 \%$ de los estudiantes lograron el puntaje de 14 (tabla 2 y grafico 2 ).

Los resultados de los estadísticos de la prueba de entrada y la prueba de salida de los estudiantes del VII y VIII semestre de la Escuela Profesional de Obstetricia, es como sigue: el promedio de la prueba de salida fue mayor que el promedio de la prueba de entrada, existiendo una diferencia de 2,1579 puntos, por otro lado el $50 \%$ de los estudiantes en la prueba de entrada lograron puntajes menores e igual a $10 \mathrm{y}$ el otro $50 \%$ de los estudiantes lograron puntajes mayores de 10 , sin embargo con respecto a la prueba de salida, el 50\% de los estudiantes alcanzaron puntajes menores e igual a 12 y el otro $50 \%$ de los estudiantes lograron puntajes mayores que 12; así mismo el valor de mayor frecuencia presentado en la prueba de entrada fue de 
10 , mientras que en la prueba de salida el valor mayor frecuencia resultó el valor de 12. Cabe manifestar que los puntajes en la prueba de entrada fueron más dispersos que los puntajes de la prueba de salida, tal como nos indica los valores de la desviación típica o estándar de ambas pruebas (tabla 3).

\section{Descripción de la población y suposiciones}

Hipótesis nula: los puntajes de la prueba de salida siguen una distribución normal,

- $¥$ hasta $+¥$

$$
\mathrm{H}_{0}: \mathrm{F}(\mathrm{x})=\mathrm{F}_{\mathrm{T}}(\mathrm{x}) \text {, para todo } \mathrm{x} \text { desde }
$$

Hipótesis alterna: los puntajes de la prueba de salida no siguen una distribución normal

Ha: $\mathrm{F}(\mathrm{x}) \neq \mathrm{F}_{\mathrm{T}}(\mathrm{x})$, para al menos un $\mathrm{x}$ En la tabla 4 se muestran los puntajes de normalidad de la prueba de Kolmogorov-Smirnov para una muestra.

Como sig. Asintot (bilateral) es menor que a $(0,000$ $<0,05$ ), en consecuencia, los puntajes de la prueba de salida siguen una distribución normal.

Contrastación de hipótesis con respecto prevención secundaria de cáncer cervical en estudiantes del VII y VIII semestre de la Escuela Profesional de obstetricia.

\section{Planteamiento de hipótesis operacional.}

$\mathrm{H}_{0}$ : No existe diferencia estadísticamente significativa de promedios en la población entre la prueba de entrada y prueba de salida en la aplicación del programa "IVAA-SCHILLER" en prevención secundaria de cáncer cervical en estudiantes del VII y VIII semestre de la Escuela Profesional de Obstetricia de la
Universidad Privada de Huancayo Franklin Roosevelt. $H_{o}: \mathrm{m}_{\mathrm{d}}=0$.

$\mathrm{H}_{\mathrm{a}}$ : Existe diferencia estadísticamente significativa de promedios en la población entre la prueba de entrada y prueba de salida en la aplicación del programa "IVAASCHILLER" en prevención secundaria de cáncer cervical en estudiantes del VII y VIII semestre de la Escuela Profesional de Obstetricia de la Universidad Privada de Huancayo Franklin Roosevelt.

$H_{a}: \mathrm{m}_{d} . \square 0$

Nivel de significación

$a=0, \sigma$

\section{Estadístico pertinente}

$\mathrm{t}$ de Student

En la tabla 5 se muestra la prueba de muestras emparejadas

\section{Decisión estadística}

Como sig bilateral es menor que $\alpha(0,000<0,05)$, entonces se rechaza la hipótesis nula (Ho) y se acepta la hipótesis alterna (Ha).

\section{Conclusión estadística}

Se concluye que: existe diferencia estadísticamente significativa de promedios en la población entre la prueba de entrada y prueba de salida en la aplicación del programa "IVAA-SCHILLER" en prevención secundaria de cáncer cervical en estudiantes del VII y VIII semestre de la Escuela Profesional de Obstetricia de la Universidad Privada de Huancayo Franklin Roosevelt.

Tabla 3. Estadísticos de la prueba de entrada y prueba de salida

\begin{tabular}{|c|c|c|c|}
\hline \multicolumn{4}{|c|}{ Estadísticos } \\
\hline & & Entrada & Salida \\
\hline \multirow{2}{*}{$\mathrm{N}$} & Válido & 57 & 57 \\
\hline & Perdidos & 0 & 0 \\
\hline \multicolumn{2}{|c|}{ Media } & 9,9825 & 12,1404 \\
\hline \multicolumn{2}{|c|}{ Mediana } & 10,0000 & 12,0000 \\
\hline \multicolumn{2}{|c|}{ Moda } & 10,00 & 12,00 \\
\hline \multicolumn{2}{|c|}{ Desv. Desviación } & 2,08302 & 1,59730 \\
\hline \multicolumn{2}{|c|}{ Varianza } & 4,339 & 2,551 \\
\hline \multicolumn{2}{|c|}{ Mínimo } & 6,00 & 10,00 \\
\hline \multicolumn{2}{|c|}{ Máximo } & 15,00 & 15,00 \\
\hline \multicolumn{2}{|c|}{ Suma } & 569,00 & 692,00 \\
\hline
\end{tabular}


Tabla 4. Normalidad de los puntajes de la prueba de salida

\begin{tabular}{llc}
\hline \multicolumn{3}{c}{ Prueba de Kolmogorov-Smirnov para una muestra } \\
\hline $\mathrm{N}$ & & Salida \\
& & 57 \\
Parámetros normales ${ }^{\mathrm{a}, \mathrm{b}}$ & Dedia & 12,1404 \\
& Absoluto & 1,59730 \\
Máximas diferencias & Positivo & 0,254 \\
extremas & Negativo & 0,254 \\
& & $-0,159$ \\
Estadístico de prueba & & 0,254 \\
Sig. asintótica(bilateral) & & $0,000^{\mathrm{c}}$ \\
\hline${ }^{\mathrm{a}}$ La distribución de prueba es normal. & \\
${ }^{\mathrm{b}}$ Se calcula a partir de datos. & \\
${ }^{\mathrm{c}}$ Corrección de significación de Lilliefors.
\end{tabular}

Tabla 5. Prueba de muestras emparejadas

\begin{tabular}{|c|c|c|c|c|c|c|c|c|c|}
\hline \multirow{3}{*}{\multicolumn{2}{|c|}{ Media }} & \multicolumn{7}{|c|}{ Diferencias emparejadas } & \multirow{3}{*}{$\begin{array}{c}\text { Sig. } \\
\text { (bilateral) }\end{array}$} \\
\hline & & \multirow[t]{2}{*}{$\begin{array}{c}\text { Desv. } \\
\text { Desviación }\end{array}$} & \multirow[t]{2}{*}{$\begin{array}{c}\text { Desv. Error } \\
\text { promedio }\end{array}$} & \multicolumn{2}{|c|}{$\begin{array}{c}95 \% \text { de intervalo } \\
\text { de confianza de la } \\
\text { diferencia }\end{array}$} & & \multirow[t]{2}{*}{$\mathbf{t}$} & \multirow[t]{2}{*}{ gl } & \\
\hline & & & & Inferior & Superior & & & & \\
\hline Par 1 & $\begin{array}{c}\text { Entrada - } \\
\text { Salida }\end{array}$ & $-2,15789$ & 2,64433 & 0,35025 & $-2,85953$ & $-1,45626$ & $-6,161$ & 56 & 0,000 \\
\hline
\end{tabular}

\section{DISCUSIÓN}

La efectividad del programa "IVAA-SCHILLER" ha dado resultados significativos en este trabajo de investigación. El estar capacitados para la detección del cáncer de cérvix a través de alternativas como el Test de IVAA y el Test de Schiller es una gran ventaja porque el diagnóstico se realiza precozmente y del mismo modo el tratamiento es oportuno y se salvan vidas valiosas.

Del mismo modo existe diferencia estadísticamente significativa de promedios en la población entre el pre test y el post test en la aplicación del programa "IVAA-SCHILLER"; por lo tanto se acepta la hipótesis alterna $(\mathrm{Ha})$; estos resultados concuerdan con la descripción que hace el Ministerio de Salud en el que el cáncer cervical patología frecuente en nuestro medio y considerado como prioridad ya que la implementación de otros métodos como screening a través de la aplicación de ácido acético llamado test de IVAA; o la aplicación del lugol llamado test de Schiller o test de IVIL; considerando que es una patología prevenible y si se diagnostica a tiempo la enfermedad puede tratarse evitándose también costos altos para el tratamiento y resultados sub óptimos con impactos negativos para nuestro país; del mismo modo el Plan Nacional de prevención y control de cáncer de cuello uterino 2017-2021 y el Instituto Nacional de Enfermedades Neoplásicas; las normas técnicas mencionan como procedimiento alternativo al Papanicolaou podemos mencionar a la inspección visual con el ácido acético y la inspección visual con el lugol; que se puede realizar en establecimientos de primer nivel, en lugares de difícil accesibilidad y en donde no se cuente con el apoyo de laboratorios de citología, realizado por profesional debidamente capacitado (obstetra); por otro lado Bellido J. en su investigación sobre onocimientos adquiridos durante el año de internado sobre prevención secundaria del cáncer de cuello uterino en estudiantes universitarios de la Escuela Académico Profesional de Obstetricia, llegando a concluir que el nivel de conocimiento adquirido durante el internado sobre prevención secundaria del cáncer de cuello uterino en estudiantes universitarios fue medio al inicio del internado con $49.2 \%$ y llegando a $52.8 \%$ al terminar el internado de tal manera que hubo un aumento de $3.6 \%$; así mismo en este estudio los resultados de los estadísticos del pre y post test aplicada a los estudiantes del VII y VIII semestre, es como sigue: el promedio del post test fue mayor que el promedio del pre test, existiendo una 
diferencia de 2,1579 puntos, por otro lado el 50\% de los estudiantes en el pre test lograron puntajes menores e igual a $10 \mathrm{y}$ el otro $50 \%$ de los estudiantes lograron puntajes mayores de 10 , sin embargo con respecto al post test, el $50 \%$ de los estudiantes alcanzaron puntajes menores e igual a 12 y el otro $50 \%$ de los estudiantes lograron puntajes mayores que 12; así mismo el valor de mayor frecuencia presentado en el pre test fue de 10, mientras que en el post test el valor mayor frecuencia resultó el valor de 12. Cabe manifestar que los puntajes en el pre test fueron más dispersos que los puntajes del post test, tal como nos indica los valores de la desviación típica o estándar de ambas pruebas.

\section{CONCLUSIONES}

Se determinó la efectividad del programa "IVAASCHILLER" en prevención secundaria de cáncer cervical en estudiantes del VII y VIII semestre de la Escuela Profesional de Obstetricia de la Universidad Privada de Huancayo Franklin Roosevelt-2021, concluyendo que existe diferencia estadísticamente significativa de promedios en la población entre la prueba de entrada y prueba de salida en la aplicación del programa "IVAA-SCHILLER"; por lo tanto se rechaza la hipótesis nula (Ho) y se acepta la hipótesis alterna (Ha).

Con respecto a la prueba de entrada y la prueba de salida de los estudiantes del VII y VIII semestre de la Escuela Profesional de Obstetricia, es como sigue: el promedio de la prueba de salida fue mayor que el promedio de la prueba de entrada, existiendo una diferencia de 2,1579 puntos, por otro lado el 50\% de los estudiantes en la prueba de entrada lograron puntajes menores e igual a $10 \mathrm{y}$ el otro $50 \%$ de los estudiantes lograron puntajes mayores de 10, sin embargo con respecto a la prueba de salida, el 50\% de los estudiantes alcanzaron puntajes menores e igual a 12 y el otro $50 \%$ de los estudiantes lograron puntajes mayores que 12; así mismo el valor de mayor frecuencia presentado en la prueba de entrada fue de 10 , mientras que en la prueba de salida el valor mayor frecuencia resultó el valor de 12. Cabe manifestar que los puntajes en la prueba de entrada fueron más dispersos que los puntajes de la prueba de salida, tal como nos indica los valores de la desviación típica o estándar de ambas pruebas.

\section{Agradecimientos}

Queremos agradecer a la Universidad Privada de Huancayo Franklin Roosevelt por darnos la oportunidad de llevar a cabo esta investigación y presentar los resultados que serán de mucho valor.

Debemos agradecer también al Centro de Salud de Palian en especial a la Obstetra Miryam Edith Davila Nuñez. por darnos las facilidades de realizar la parte práctica de las capacitaciones del programa "IVAASCHILLER"para las estudiantes de obstetricia del VII y VIII semestre.

\section{Correspondencia:}

Liz Miriam Suárez Reynoso

suarezreynoso@uroosevelt.edu.pe

\section{REFERENCIAS BIBLIOGRÁFICAS}

1. Organización Mundial de la Salud. Control integral del cáncer cervico uterino. Guía de prácticas esenciales. Washington DC Organización Mundial de la Salud. (Citado el 15 de febrero del 2021) Disponible en: http://iris.paho.org/xmlui/ bitstream/handl/123456789/28512/9789275318799 spa.pdf?ua $=1$

2. Bray F, Ferlay J, Soerjomataran I, Siegel R, Torre L, Jemal A. Global cancer statistics 2018: GLOBOCAN estimates of incidence and mortality worldwide for 36 cancers in 185 countries. CA Cancer J Clin. 2018;68(6):394-424. doi: 10.3322/caac.21492

3. Ministerio de Salud. Plan Nacional de prevención y control de cáncer de cuello uterino 2017-2021( R.M.N440-2017/MINSA). Lima, Perú: Ministerio de Salud; 2021. (Citado el 15 de febrero del 2021) Disponible en: https://www.gob.pe/institucion/minsa/ informes-publicaciones/280845-plan-nacional-parala-prevencion-y-control-de-cancer-de-cuello-uterino2017-2021-r-m-n-440-2017-minsa.

4. Instituto Nacional de Enfermedades Neoplásicas. Norma técnico-oncológica para la prevención, detección y manejo de las lesiones pre malignas del cuello uterino a nivel nacional. Lima: Instituto Nacional de Enfermedades Neoplásicas. (Citado el 15 de febrero del 2021) Disponible en: http://bvs. minsa.gob.pe/local/MINSA/786_MINSA1091.pdf

5. Sellors J. La colposcopia y el tratamiento de la neoplasia intraepitelial cervical. (Citado el 15 de febrero del 2021) Disponible en: https://screening. iarc.fr/doc/colpoesmanual.pdf

6. Bellido J. Conocimientos adquiridos durante el año de internado sobre prevención secundaria del cáncer de cuello uterino en estudiantes universitarios de la Escuela Académico Profesional de Obstetricia. Tesis. Lima: Universidad Nacional Mayor de San Marcos; 2014. (Citado el 15 de febrero del 2021) Disponible en: https://cybertesis.unmsm. edu.pe/bitstream/handle/20.500.12672/4053/ 
Bellido_mj.pdf?sequence $=1 \&$ isAllowed $=\mathrm{y}$

7. Del Valle A. Inspección Visual con Ácido Acético (IVAA) en la detección precoz del cáncer de cuello uterino-Estudio Comparativo. Tesis para optar al Título de Doctor en Medicina y Cirugía. Cordoba, Argentina: Universidad Nacional de Córdoba; 2019. (Citado el 15 de febrero del 2021) Disponible en: http://lildbi.fcm.unc.edu.ar/lildbi/tesis/foresi_ana_ maria_valle.pdf

8. Organización Panamericana de la Salud. Control integral del cáncer cervico uterino. Guía de prácticas esenciales. Washington DC: Organización
Panamericana de la Salud. (Citado el 15 de febrero del 2021) Disponible en: http://iris.paho.org/xmlui/ bitstream/handl/123456789/28512/9789275318799 spa.pdf?ua $=1$

9. Escuela Latinoamericana de Cáncer de Cérvix. Manual de referencia del curso clínico de Prevención Secundaria en Cáncer de Cuello Uterino basado en la Inspección Visual con Ácido Acético y Crioterapia. Escuela Latinoamericana de Cáncer de Cérvix; 2012.

Recibido: 04/04/2021

Aceptado: 18/10/2021 